\title{
Vibrational analysis of deoxy-andrographolide using MM/QM methods
}

\author{
P.K. Singh a , Tanveer Hasan ${ }^{\text {a }}$, Onkar Prasad ${ }^{\text {a }}$, Leena Sinha ${ }^{\text {a }}$, Sudha Jain ${ }^{\text {b }}$, Kanwal Raj ${ }^{\text {c,* }}$, \\ N. Sundaraganesan ${ }^{\mathrm{d}}$ and Neeraj Misra ${ }^{\mathrm{a}, * *}$ \\ ${ }^{a}$ Department of Physics, Lucknow University, 226007, India \\ ${ }^{\mathrm{b}}$ Department of Chemistry, Lucknow University, 226007, India \\ ${ }^{\mathrm{c}}$ Medicinal and Process Chemistry Division, Central Drug Research Institute, Lucknow 226001, India \\ ${ }^{\mathrm{d}}$ Department of Physics (Engg), Annamalai University, Annamalainagar 608002, India
}

\begin{abstract}
Bioactive natural products have an enormous economic importance as speciality chemicals. Extracts of the shrub Andrographis paniculata are widely used as herbal medicines in many countries. Diterpenoid lactones isolated from these extracts have been the subject of extensive investigation, and one of its constituent diterpenoid is deoxy-andrographolide. A complete normal coordinate analysis was performed for deo-andrographolide in terms of the calculation by using Classical mechanics and Quantum mechanical methods. The study of vibrational spectra helps in understanding the dynamical behaviour and hence a lot of information can be derived about the conformation. The geometrical parameters and the vibrational spectra have been discussed in detail.
\end{abstract}

Keywords: FT-IR spectra, lactones, normal mode analysis, MM, Hartree-Fock

\section{Introduction}

Natural products offer unmatched structural variety, especially as new environmental niches are explored, and their usefulness can be further extended by engineering the proteins that produce them and using them to probe biological pathways [1]. Rediscovery of the connection between plants and health is responsible for launching a new generation of botanical therapeutics that include plant derived therapeutics. Natural products evolved to perform a function that is achieved by binding to proteins. Therefore, natural product should be able to penetrate the biological barrier and make their ways to certain cells or organs in which they will exert their effect. Thus, natural product is already validated to reach and bind specific proteins. The similarity in protein domain structure can be exploited to develop inhibitors of enzymes with different functions based on the guiding structure of a natural product.

Natural compounds contains a range of complex organic chemicals that may have synergistic effect on various physiological process in normal and disease state by interacting with multiple metabolic pathways [2]. Andrographis paniculata, a medicinal herb is reported to have antidiabetic [3] and antithrombotic [4] effects. It belongs to the family Acanthaceae and is a rich source of many bioactive

\footnotetext{
${ }^{*}$ Responsible for experimental chemistry.

** Corresponding author: N. Misra, B-981, sector-A, Mahanagar, Lucknow 226006, India. E-mail: neerajmisra@ hotmail.com.
} 
compounds. Its antioxidant and antiinflammory activity has already been reported [5]. Major active component of this plant andrographolide has shown to possess pharmacological properties which encompass antihepatotoxic [6] and antiviral effects [7]. It has recently been reported that andrographolide inhibits NF- $k \mathrm{~B}$ binding to DNA and thus reducing the expression of proinflammatory proteins such as cycloxygenase-2 (COX-2) [8].

The present work on the diterpenoid "deoxy-andrographolide" is in continuation to our earlier work on andrographolide [9] and neo-andrographolide [10]. Since this constituent of Andrographis paniculata (AP) has been identified as scaffold for the generation of combinatorial libraries using solid phase methods [11], it was decided to study its molecular structure and vibrational spectra in detail. This compound has also been widely used for the treatment of calcium channel blocking activity, platelet activating factor (PAF) antagonistic activity, anti-inflammatory activity, anti-cancer activity apart from many other like analgesic, antipyretic, anti ulcerogenic effects and hence it has aroused the interest of researchers to probe in detail its structure and dynamics [12-14].

To the best of our knowledge no study on the normal coordinate analysis on deoxy-andrographolide has been reported so far in the literature apart from few spectroscopic studies [15,16]. As a part of our ongoing research work $[17,18]$ on vibrational analysis in a variety of macromolecules, here in the present communication, the experimental FT-IR frequencies of the title molecule deoxy-andrographolide are compared with theoretical frequencies obtained by normal coordinate analysis using two different methods: a classical mechanics method and the Ab initio Hartree-Fock method. Thus the evaluation of the advantages of both the methods is useful for obtaining a reliable assignment of the vibrational spectra. The purpose of this study assumes importance because of our earlier work [9] on the major constituent of AP i.e. andrographolide and hence a comparative study between the two diterpenes can be carried out.

\section{Computational methods}

\subsection{Classical mechanics calculations}

The normal coordinate analysis is based on the Wilson GF matrix method [19-21] employing Urey Bradley force field [22] to evaluate normal modes. The Cartesian coordinates were calculated from X-ray crystallographic data obtained by Rajnikant et al. [23]. The title compound Deoxy-andrographolide, a diterpenoid, crystallizes in the monoclinic space group $\mathrm{P}_{21}$. The six membered rings are trans-fused and both are in the chair conformation. The furan ring is planar. Molecules are held together by $\mathrm{C}-\mathrm{H}$. . O and O-H. . .O hydrogen bonds. Basak et al. [24,25] have shown that andrographolide could be considered as an important precursor structure that might require further structure modification for enhancement of its enzyme inactivation property. The modification of hydroxyl groups in Andrographis paniculata could lead to a better understanding of its inhibitory action. The actual mechanism by which these diterpenes exert their inhibitory action against convertases is not currently understood and that the molecule contains a highly reactive five membered lactone ring.

\subsection{Quantum mechanical calculations}

The entire calculations involving Hartree-Fock (HF) level were performed on the Pentium IV/2.9 GHz personal computer using Gaussian $03 \mathrm{~W}$ program package [26], invoking gradient geometry optimization 
[27]. Initial geometry generated from standard geometrical parameters was minimized without any constraint in the potential energy surface at Hartree-Fock level, adopting the standard 3-21 basis set. In order to obtain a more complete description of the molecular motion the vibrational frequency calculations were carried out at HF level. Then vibrationally averaged nuclear positions of Deoxy-andrographolide were used for harmonic vibrational frequency calculation resulting in IR frequencies.

By combining the result of GaussView program [28] with symmetry considerations, vibrational frequency assignments were made with a high degree of accuracy. The defined coordinates form a complete set and matches well with the motions observed using the GaussView program.

\section{Experimental}

The FTIR spectra of Deoxy-andrographolide have been recorded in CsI on a Perkin Elmer 1800 spectrophotometer. Spectroscopic preparation of sample were carried out under an atmosphere of prepurified nitrogen. Deoxy-andrographolide was isolated from fresh plant material of Andrographis paniculata to the procedure reported in literature [29]. The compound was identified by comparison with its IR, MS, NMR data with that of reported in literature $[29,30]$.

Isolation: plant material (Andrographis paniculata, $1 \mathrm{~kg}$ ) was powdered and percolated with ethanol $(3 \times 51)$. The ethanolic extractwas concentrated in vacuo at $50^{\circ} \mathrm{C}$. This was diluted with water $(750 \mathrm{ml})$ and fractionated to hexane $(9 \mathrm{~g})$, chloroform $(10.6 \mathrm{~g})$, butanol $(30 \mathrm{~g})$ and aquous fractions $(25 \mathrm{~g})$. The butanol fraction ( $10 \mathrm{~g}$ ) was chromatographed over a column of silica gel (300 g) in chloroform and eluted with chloroform, chloroform: ethylacetate, ethylacetate, ethylacetate: methanol, chloroform: ethylacetate eluate was further purified by rechromatography and crystallization with chloroform gave deoxyandrographolide (400 mg, 0.04\%): m.p. $169^{\circ} \mathrm{C}$. Mass spectrum (FAB) $m / z 335,357\left(\mathrm{M}^{+}+\mathrm{Na}\right)$; ${ }^{1} \mathrm{H} N M R\left(400 \mathrm{MHz}, \mathrm{CDCl}_{3}\right): 7.10$ (brs, 1H, H-14), 4.89 (s, 1H, H-17), 4.78 (s, 2H, H-15), 4.60 (s, 1H, $\mathrm{H}-17), 4.16(\mathrm{~d}, 12,1 \mathrm{H}, \mathrm{H}-19), 3.48$ (t, 6, 1H, H-3), 3.31 (d, 12, 1H, H-19), 2.40-2.50 (m, 2H, H-9 \& $\mathrm{H}-7), 2.00-1.00\left(\mathrm{~m}, 12 \mathrm{H}, 6 \times \mathrm{CH}_{2}\right), 1.21$ and $0.70(2 \mathrm{~s}, 3 \mathrm{H}$ each, $\mathrm{H}-20 \& \mathrm{H}-18)$.

\section{Result and discussion}

\subsection{Vibrational investigation}

\subsubsection{Molecular mechanics calculations}

Normal coordinate calculations were performed using the Shimanouchi program [31] which follows the Wilson's G-F matrix method. This method describes the motion in terms of the internal coordinates, which are changes in bond lengths, bond angles and those out of plane bending and dihedral angles. The force constants in terms of these coordinates can be easily visualized and have a physical meaning. We have used a Urey-Bradley force field in our calculations. It incorporates intra unit interactions and interactions due to the neighboring units, in addition to the bonded interactions. It also includes the interactions between non-bonded atoms. Force constants were initially taken from the literature [32] and later modified to give the "best fit" with the observed FT-IR spectra.

The Deoxy-andrographolide has 54 atoms, however to reduce the problem to manageable dimensions $\mathrm{CH}, \mathrm{CH}_{2}, \mathrm{CH}_{3}$ have been treated as mass points with a mass of 13,14 and 15 respectively. This does not in any way disturb the accuracy of the results reported here. This is because the frequencies belonging to these can be well designated as group frequencies and many of them being in the higher range do not mix 
with other modes. With this approximation the andrographolide problem reduces to 24 atom problem with 66 normal modes of vibrations. The structural data used for Deoxy-andrographolide is reported by Rajnikant et al. [23]. The model molecular structure of deoxy-andrographolide used in normal coordinate analysis is given in Fig 1. The internal coordinates and force constants for deoxy-andrographolide are given in Table 1. The final force constant values for the corresponding modes are given in Table 2.

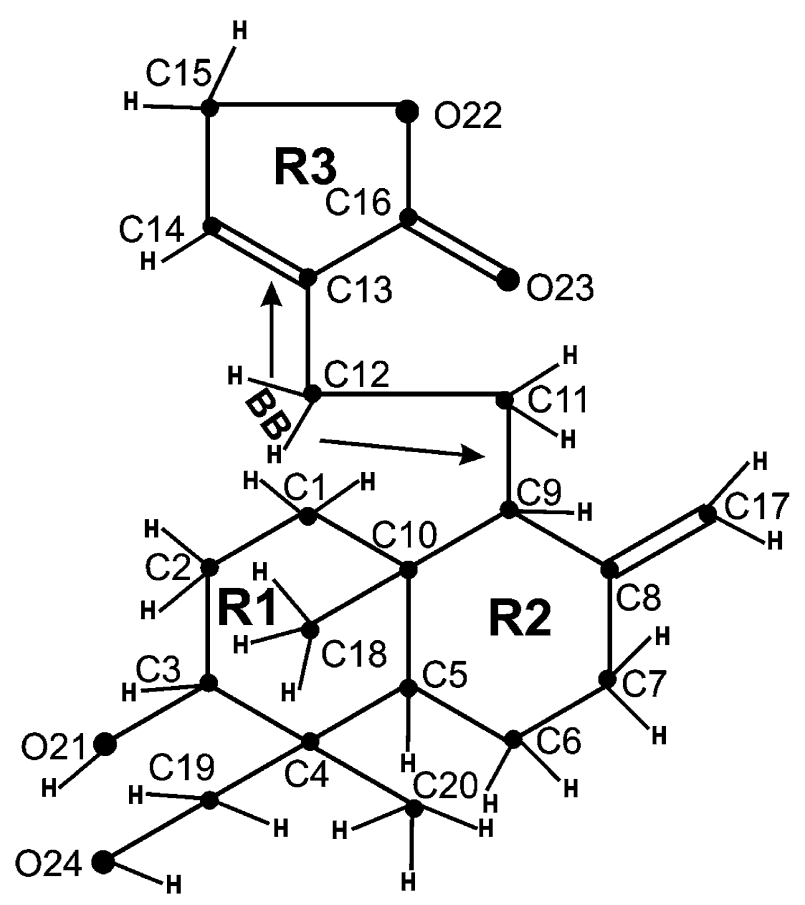

Fig. 1. Model molecular structure of 14-deoxyandrographolide.

Table 1

Internal co-ordinates and Urey-Bradley force constants (md/Å)

\begin{tabular}{|c|c|c|c|}
\hline Internal coordinate & Force constant & Internal coordinate & Force constant \\
\hline 1. $\quad \nu(\mathrm{C} 1-\mathrm{C} 2)$ & 3.300 & 47. $\Phi(\mathrm{C} 8-\mathrm{C} 9-\mathrm{C} 11)$ & $0.460(0.420)$ \\
\hline 2. $\quad \nu(\mathrm{C} 1-\mathrm{C} 10)$ & 3.300 & 48. $\Phi(\mathrm{C} 10-\mathrm{C} 9-\mathrm{C} 11)$ & $0.460(0.420)$ \\
\hline 3. $\nu(\mathrm{C} 2-\mathrm{C} 3)$ & 3.300 & 49. $\Phi(\mathrm{C} 1-\mathrm{C} 10-\mathrm{C} 5)$ & $0.880(0.850)$ \\
\hline 4. $\nu(\mathrm{C} 3-\mathrm{C} 4)$ & 3.300 & 50. $\Phi(\mathrm{C} 1-\mathrm{C} 10-\mathrm{C} 9)$ & $0.560(0.700)$ \\
\hline 5. $\nu(\mathrm{C} 3-\mathrm{O} 21)$ & 4.800 & 51. $\Phi(\mathrm{C} 1-\mathrm{C} 10-\mathrm{C} 18)$ & $0.460(0.400)$ \\
\hline 6. $\nu(\mathrm{C} 4-\mathrm{C} 5)$ & 3.300 & 52. $\Phi(\mathrm{C} 5-\mathrm{C} 10-\mathrm{C} 9)$ & $0.410(0.400)$ \\
\hline 7. $\nu(\mathrm{C} 4-\mathrm{C} 19)$ & 4.100 & 53. $\Phi(\mathrm{C} 5-\mathrm{C} 10-\mathrm{C} 18)$ & $0.560(0.500)$ \\
\hline 8. $\nu(\mathrm{C} 4-\mathrm{C} 20)$ & 5.130 & 54. $\Phi(\mathrm{C} 9-\mathrm{C} 10-\mathrm{C} 18)$ & $0.760(0.700)$ \\
\hline 9. $\nu(\mathrm{C} 5-\mathrm{C} 6)$ & 3.200 & 55. $\Phi(\mathrm{C} 9-\mathrm{C} 11-\mathrm{C} 12)$ & $0.980(0.980)$ \\
\hline 10. $\quad \nu(\mathrm{C} 5-\mathrm{C} 10)$ & 4.300 & 56. $\Phi(\mathrm{C} 11-\mathrm{C} 12-\mathrm{C} 13)$ & $0.980(0.900)$ \\
\hline 11. $\nu(\mathrm{C} 6-\mathrm{C} 7)$ & 3.200 & 57. $\Phi(\mathrm{C} 12-\mathrm{C} 13=\mathrm{C} 14)$ & $1.400(0.750)$ \\
\hline 12. $\nu(\mathrm{C} 7-\mathrm{C} 8)$ & 3.200 & 58. $\Phi(\mathrm{C} 12-\mathrm{C} 13-\mathrm{C} 16)$ & $0.560(0.560)$ \\
\hline 13. $\nu(\mathrm{C} 8-\mathrm{C} 9)$ & 3.200 & 59. $\Phi(\mathrm{C} 14=\mathrm{C} 13-\mathrm{C} 16)$ & $0.760(0.590)$ \\
\hline 14. $\nu(\mathrm{C} 8=\mathrm{C} 17)$ & 9.410 & 60. $\Phi(\mathrm{C} 13=\mathrm{C} 14-\mathrm{C} 15)$ & $0.760(0.590)$ \\
\hline 15. $\nu(\mathrm{C} 9-\mathrm{C} 10)$ & 3.200 & 61. $\Phi(\mathrm{C} 14-\mathrm{C} 15-\mathrm{C} 22)$ & $0.530(0.520)$ \\
\hline
\end{tabular}


Table 1

(Continued)

\begin{tabular}{|c|c|c|c|}
\hline Internal coordinate & Force constant & Internal coordinate & Force constant \\
\hline 16. $\quad \nu(\mathrm{C} 9-\mathrm{C} 11)$ & 5.650 & 62. $\Phi(\mathrm{C} 13-\mathrm{C} 16-\mathrm{O} 22)$ & $0.530(0.520)$ \\
\hline 17. $\nu(\mathrm{C} 10-\mathrm{C} 18)$ & 4.748 & 63. $\Phi(\mathrm{C} 13-\mathrm{C} 16=\mathrm{O} 23)$ & $1.550(0.760)$ \\
\hline 18. $\nu(\mathrm{C} 11-\mathrm{C} 12)$ & 4.600 & 64. $\Phi(\mathrm{C} 22-\mathrm{C} 16=\mathrm{O} 23)$ & $0.860(0.560)$ \\
\hline 19. $\quad \nu(\mathrm{C} 12-\mathrm{C} 13)$ & 5.390 & 65. $\Phi(\mathrm{C} 4-\mathrm{C} 19-\mathrm{O} 24)$ & $0.580(0.650)$ \\
\hline 20. $\quad \nu(\mathrm{C} 13=\mathrm{C} 14)$ & 6.890 & 66. $\Phi(\mathrm{C} 15-\mathrm{O} 22-\mathrm{C} 16)$ & $1.200(0.760)$ \\
\hline 21. $\nu(\mathrm{C} 13-\mathrm{C} 16)$ & 4.645 & 67. $\omega(\mathrm{C} 17=\mathrm{C} 18)$ & 0.215 \\
\hline 22. $\nu(\mathrm{C} 14-\mathrm{C} 15)$ & 4.645 & 68. $\omega(\mathrm{C} 18-\mathrm{C} 10)$ & 0.215 \\
\hline 23. $\nu(\mathrm{C} 15-\mathrm{O} 22)$ & 5.000 & 69. $\omega(\mathrm{C} 20-\mathrm{C} 4)$ & 0.225 \\
\hline 24. $\quad \nu(\mathrm{C} 16-\mathrm{O} 22)$ & 5.300 & 70. $\omega(\mathrm{O} 21-\mathrm{C} 3)$ & 0.225 \\
\hline 25. $\nu(\mathrm{C} 16=\mathrm{O} 23)$ & 8.352 & 71. $\omega(\mathrm{O} 23=\mathrm{C} 16)$ & 0.225 \\
\hline 26. $\nu(\mathrm{C} 19=\mathrm{O} 24)$ & 6.410 & 72. $\tau(\mathrm{C} 1-\mathrm{C} 2)$ & 0.025 \\
\hline 27. $\Phi(\mathrm{C} 2-\mathrm{C} 1-\mathrm{C} 10)$ & $0.880(0.850)$ & 73. $\tau(\mathrm{C} 1-\mathrm{C} 10)$ & 0.025 \\
\hline 28. $\Phi(\mathrm{C} 1-\mathrm{C} 2-\mathrm{C} 3)$ & $0.880(0.850)$ & 74. $\tau(\mathrm{C} 2-\mathrm{C} 3)$ & 0.025 \\
\hline 29. $\Phi(\mathrm{C} 2-\mathrm{C} 3-\mathrm{C} 4)$ & $0.880(0.850)$ & 75. $\tau(\mathrm{C} 3-\mathrm{C} 4)$ & 0.025 \\
\hline 30. $\Phi(\mathrm{C} 2-\mathrm{C} 3-\mathrm{C} 21)$ & $0.810(0.760)$ & 76. $\tau(\mathrm{C} 4-\mathrm{C} 5)$ & 0.025 \\
\hline 31. $\Phi(\mathrm{C} 4-\mathrm{C} 3-\mathrm{O} 21)$ & $0.810(0.760)$ & 77. $\tau(\mathrm{C} 4-\mathrm{C} 19)$ & 0.025 \\
\hline 32. $\Phi(\mathrm{C} 3-\mathrm{C} 4-\mathrm{C} 5)$ & $0.880(0.850)$ & 78. $\tau(\mathrm{C} 5-\mathrm{C} 6)$ & 0.025 \\
\hline 33. $\Phi(\mathrm{C} 3-\mathrm{C} 4-\mathrm{C} 19)$ & $0.460(0.400)$ & 79. $\tau(\mathrm{C} 5-\mathrm{C} 10)$ & 0.022 \\
\hline 34. $\Phi(\mathrm{C} 3-\mathrm{C} 4-\mathrm{C} 20)$ & $0.460(0.450)$ & 80. $\tau(\mathrm{C} 6-\mathrm{C} 7)$ & 0.025 \\
\hline 35. $\Phi(\mathrm{C} 5-\mathrm{C} 4-\mathrm{C} 19)$ & $0.460(0.400)$ & 81. $\tau(\mathrm{C} 7-\mathrm{C} 8)$ & 0.025 \\
\hline 36. $\Phi(\mathrm{C} 5-\mathrm{C} 4-\mathrm{C} 20)$ & $0.460(0.450)$ & 82. $\tau(\mathrm{C} 8-\mathrm{C} 9)$ & 0.025 \\
\hline 37. $\Phi(\mathrm{C} 19-\mathrm{C} 4-\mathrm{C} 20)$ & $0.660(0.560)$ & 83. $\tau(\mathrm{C} 9-\mathrm{C} 10)$ & 0.025 \\
\hline 38. $\Phi(\mathrm{C} 4-\mathrm{C} 5-\mathrm{C} 6)$ & $0.560(0.700)$ & 84. $\tau(\mathrm{C} 9-\mathrm{C} 11)$ & 0.025 \\
\hline 39. $\Phi(\mathrm{C} 4-\mathrm{C} 5-\mathrm{C} 10)$ & $0.880(0.850)$ & 85. $\tau(\mathrm{C} 11-\mathrm{C} 12)$ & 0.025 \\
\hline 40. $\Phi(\mathrm{C} 6-\mathrm{C} 5-\mathrm{C} 10)$ & $0.410(0.400)$ & 86. $\tau(\mathrm{C} 12-\mathrm{C} 13)$ & 0.025 \\
\hline 41. $\Phi(\mathrm{C} 5-\mathrm{C} 6-\mathrm{C} 7)$ & $0.410(0.400)$ & 87. $\tau(\mathrm{C} 13-\mathrm{C} 14)$ & 0.022 \\
\hline 42. $\Phi(\mathrm{C} 6-\mathrm{C} 7-\mathrm{C} 8)$ & $0.410(0.400)$ & 88. $\tau(\mathrm{C} 13-\mathrm{C} 16)$ & 0.018 \\
\hline 43. $\Phi(C 7-C 8-C 9)$ & $0.410(0.400)$ & 89. $\tau(\mathrm{C} 14-\mathrm{C} 15)$ & 0.018 \\
\hline 44. $\Phi(\mathrm{C} 7-\mathrm{C} 8=\mathrm{C} 17)$ & $0.880(0.940)$ & 90. $\tau(\mathrm{C} 15-\mathrm{O} 22)$ & 0.017 \\
\hline 45. $\Phi(\mathrm{C} 9-\mathrm{C} 8=\mathrm{C} 17)$ & $0.880(0.940$ & 91. $\tau(\mathrm{C} 16-\mathrm{C} 22)$ & 0.018 \\
\hline 46. $\Phi(\mathrm{C} 8-\mathrm{C} 9=\mathrm{C} 10)$ & $0.410(0.400)$ & & \\
\hline
\end{tabular}

$\nu(\mathrm{X}-\mathrm{Y})$ represents the stretching between the atoms $\mathrm{X}$ and $\mathrm{Y}$ etc.

$\Phi(X-Y-Z)$ represents the in plane bending between the atoms $X, Y$ and $Z$.

$\omega(\mathrm{X}-\mathrm{Y})$ represents the out of plane bending (wagging) between the atoms $\mathrm{X}$ and $\mathrm{Y}$.

$\tau(\mathrm{X}-\mathrm{Y})$ represents the torsion of the atoms $\mathrm{X}$ and $\mathrm{Y}$.

The number in parenthesis represents the non-bonded value of the atoms $\mathrm{X}, \mathrm{Y}$ and $\mathrm{Z}$.

The frequencies along with the potential energy distribution are given in Table 3. The FT-IR spectra of Deoxy-andrographolide is given in Fig 2. The observed frequencies agree with the calculated ones within $5 \mathrm{~cm}^{-1}$. In the assignment of the normal modes only the dominant potential energy distributions are given. Identification with the experimental data has been made on the basis of potential energy distribution, line profile, relative intensities, energies and the presence/absence of a given mode in similar molecules.

Although a number of spectroscopic studies [32,33] have been performed on andrographis paniculata but presumably, the data for the normal mode analysis on deoxy-andrographolide is being reported for 
Table 2

Force constant values for corresponding modes

\begin{tabular}{|c|c|c|}
\hline S. no & Modes & Force constant values \\
\hline 1 & $\mathrm{v}(\mathrm{C}-\mathrm{C}) \mathrm{R} 1$ & 3.3000 \\
\hline 2 & $\mathrm{v}(\mathrm{C}-\mathrm{C}) \mathrm{R} 2$ & 3.2000 \\
\hline 3 & $\mathrm{v}(\mathrm{C}-\mathrm{C}) \mathrm{R} 3$ & 4.6450 \\
\hline 4 & $\mathrm{v}(\mathrm{C}=\mathrm{C}) \mathrm{R} 3$ & 6.8900 \\
\hline 5 & $\mathrm{v}(\mathrm{C}-\mathrm{C}) \mathrm{cm} \mathrm{R} 1 \& \mathrm{R} 2$ & 4.3000 \\
\hline 6 & $\mathrm{v}(\mathrm{C}-\mathrm{O})$ adj $\mathrm{R} 1$ & 4.8000 \\
\hline 7 & $\mathrm{v}(\mathrm{C}-\mathrm{C}) \operatorname{adj} \mathrm{R} 1$ link $\mathrm{O}$ & 4.1000 \\
\hline 8 & $\mathrm{v}(\mathrm{C}-\mathrm{C})$ adj $\mathrm{R} 1$ & 5.1300 \\
\hline 9 & $\mathrm{v}(\mathrm{C}=\mathrm{C})$ adj $\mathrm{R} 2$ & 9.4100 \\
\hline 10 & $\mathrm{v}(\mathrm{C}-\mathrm{C})$ adj $\mathrm{R} 2$ & 5.6500 \\
\hline 11 & $\mathrm{v}(\mathrm{C}-\mathrm{C})$ adj cm R1\&R2 & 4.7480 \\
\hline 12 & $\mathrm{v}(\mathrm{C}-\mathrm{C}) \mathrm{BB}$ & 4.6000 \\
\hline 13 & $\mathrm{v}(\mathrm{C}-\mathrm{C})$ adj $\mathrm{R} 3$ & 5.3900 \\
\hline 14 & $\mathrm{v}(\mathrm{C}-\mathrm{O}) \mathrm{R} 3$ & 5.0000 \\
\hline 15 & $\mathrm{v}(\mathrm{C}-\mathrm{O}) \mathrm{R} 3 \operatorname{adj}(=\mathrm{O})$ & 5.3000 \\
\hline 16 & $\mathrm{v}(\mathrm{C}=\mathrm{O})$ adj $\mathrm{R} 3$ & 8.3520 \\
\hline 17 & $\mathrm{v}(\mathrm{C}-\mathrm{O})$ link $\mathrm{R} 1$ & 6.4100 \\
\hline 18 & $\mathrm{a}(\mathrm{C}-\mathrm{C}-\mathrm{C}) \mathrm{R} 1$ & 0.8800 \\
\hline 19 & $\mathrm{a}(\mathrm{C}-\mathrm{C}-\mathrm{C}) \mathrm{R} 2$ & 0.4100 \\
\hline 20 & $\mathrm{a}(\mathrm{C}=\mathrm{C}-\mathrm{C}) \mathrm{R} 3$ & 0.7600 \\
\hline 21 & $\mathrm{a}(\mathrm{C}-\mathrm{C}-\mathrm{C})$ adj $\mathrm{R} 1 \ln \mathrm{O}$ & 0.4600 \\
\hline 22 & $\mathrm{a}(\mathrm{C}-\mathrm{C}-\mathrm{C}) \mathrm{o} / \mathrm{s} \mathrm{R} 2$ & 0.6600 \\
\hline 23 & $\mathrm{a}(\mathrm{O}-\mathrm{C}-\mathrm{C}) \mathrm{cm} \mathrm{R} 1 \& \mathrm{R} 2$ & 0.5600 \\
\hline 24 & $\mathrm{a}(\mathrm{C}-\mathrm{C}=\mathrm{C})$ adj $\mathrm{R} 2$ & 0.8800 \\
\hline 25 & $\mathrm{a}(\mathrm{C}-\mathrm{C}-\mathrm{C})$ adj $\mathrm{R} 2 \ln \mathrm{BB}$ & 0.4600 \\
\hline 26 & $\mathrm{a}(\mathrm{C}-\mathrm{C}-\mathrm{C})$ adj $\mathrm{R} 1$ ter & 0.4601 \\
\hline 27 & $\mathrm{a}(\mathrm{C}-\mathrm{C}-\mathrm{C}) \mathrm{cm} \mathrm{R} 1 \& \mathrm{R} 2$ & 0.5600 \\
\hline 28 & $\mathrm{a}(\mathrm{C}-\mathrm{C}-\mathrm{C})$ adj $\mathrm{R} 2$ & 0.7600 \\
\hline 29 & $\mathrm{a}(\mathrm{C}-\mathrm{C}-\mathrm{C}) \mathrm{R} 2 \ln \mathrm{BB}$ & 0.9800 \\
\hline 30 & $\mathrm{a}(\mathrm{C}-\mathrm{C}-\mathrm{C}) \mathrm{BB}$ & 0.9800 \\
\hline 31 & $\mathrm{a}(\mathrm{C}-\mathrm{C}=\mathrm{C})$ adj $\mathrm{R} 3$ & 1.4000 \\
\hline 32 & $\mathrm{a}(\mathrm{C}-\mathrm{C}-\mathrm{C})$ adj $\mathrm{R} 3$ & 0.5600 \\
\hline 33 & $\mathrm{a}(\mathrm{C}-\mathrm{C}-\mathrm{O}) \mathrm{R} 3$ & 0.5300 \\
\hline 34 & $\mathrm{a}(\mathrm{C}-\mathrm{C}=\mathrm{O})$ adj $\mathrm{R} 3$ & 1.5500 \\
\hline 35 & $\mathrm{a}(\mathrm{O}-\mathrm{C}=\mathrm{O})$ adj $\mathrm{R} 3$ & 0.8600 \\
\hline 36 & $\mathrm{a}(\mathrm{C}-\mathrm{C}-\mathrm{O})$ adj $\mathrm{R} 1 \ln \mathrm{O}$ & 0.5800 \\
\hline 37 & $\mathrm{a}(\mathrm{C}-\mathrm{O}-\mathrm{C}) \mathrm{R} 3$ & 1.2000 \\
\hline 38 & $\mathrm{a}(\mathrm{C}-\mathrm{C}-\mathrm{O})$ adj $\mathrm{R} 1$ & 0.8100 \\
\hline 39 & $\mathrm{w}(\mathrm{C}-\mathrm{C}) \mathrm{R} 2$ & 0.2150 \\
\hline 40 & $\mathrm{w}(\mathrm{C}-\mathrm{C}) \mathrm{cm} \mathrm{R} 1 \& \mathrm{R} 2$ & 0.2150 \\
\hline 41 & $\mathrm{w}(\mathrm{C}-\mathrm{C}) \mathrm{R} 1$ & 0.2250 \\
\hline 42 & $\mathrm{w}(\mathrm{C}-\mathrm{C}) \mathrm{R} 1$ & 0.2250 \\
\hline 43 & $\mathrm{w}(\mathrm{O}-\mathrm{C}) \mathrm{R} 3$ & 0.2250 \\
\hline 44 & $\mathrm{t}(\mathrm{C}-\mathrm{C}) \mathrm{R} 1$ & 0.0250 \\
\hline 45 & $\mathrm{t}(\mathrm{C}-\mathrm{C}) \operatorname{adj} \mathrm{R} 1$ & 0.0250 \\
\hline
\end{tabular}


Table 2

(Continued)

\begin{tabular}{llc}
\hline S. no & \multicolumn{1}{c}{ Modes } & Force constant values \\
\hline 46 & $\mathrm{t}(\mathrm{C}-\mathrm{C}) \mathrm{R} 2$ & 0.0250 \\
47 & $\mathrm{t}(\mathrm{C}-\mathrm{C})$ adj $\mathrm{R} 2$ & 0.0250 \\
48 & $\mathrm{t}(\mathrm{c}-\mathrm{C}) \mathrm{BB}$ & 0.0250 \\
49 & $\mathrm{t}(\mathrm{C}-\mathrm{C})$ adj R3 & 0.0250 \\
50 & $\mathrm{t}(\mathrm{C}=\mathrm{C}) \mathrm{R} 3$ & 0.0220 \\
51 & $\mathrm{t}(\mathrm{C}-\mathrm{C}) \mathrm{R} 3$ & 0.0180 \\
52 & $\mathrm{t}(\mathrm{C}-\mathrm{O}) \mathrm{R} 3$ & 0.0170 \\
53 & $\mathrm{t}(\mathrm{C}=\mathrm{O})$ adj R3 & 0.0180 \\
54 & $\mathrm{t}(\mathrm{C}-\mathrm{C})$ cm R1\&R2 & 0.0220
\end{tabular}

Abbreviations used in the table have the following meanings: $\mathrm{R}$ - ring; adj - adjacent to; $\mathrm{BB}$ - back bone; $\mathrm{cm}$ - common to; ln - linking; o/s - outside.

Table 3

\begin{tabular}{|c|c|c|}
\hline $\begin{array}{l}\text { Calculated freq. } \\
\qquad\left(\mathrm{cm}^{-1}\right)\end{array}$ & $\begin{array}{l}\text { Observed freq. } \\
\qquad\left(\mathrm{cm}^{-1}\right)\end{array}$ & Assignment (\% potential energy distribution) \\
\hline 1754 & 1754 & $\nu(\mathrm{C}=\mathrm{O})$ adj $\mathrm{R} 3[51]+\nu(\mathrm{C}-\mathrm{C}) \mathrm{R} 3[41]+\nu(\mathrm{C}-\mathrm{C})$ adj $\mathrm{R} 3$ [9] \\
\hline 1697 & 1700 & $\nu(\mathrm{C}=\mathrm{C})$ adj $\mathrm{R} 2[96]+\nu(\mathrm{C}-\mathrm{C}) \mathrm{R} 2[11]$ \\
\hline 1632 & 1636 & $\nu(\mathrm{C}=\mathrm{C}) \mathrm{R} 3[63]+\nu(\mathrm{C}-\mathrm{C})$ adj $\mathrm{R} 3[28]+\nu(\mathrm{C}-\mathrm{C}) \mathrm{R} 3[5]$ \\
\hline 1594 & 1595 & $\nu(\mathrm{C}-\mathrm{C})$ adj $\mathrm{R} 2[44]+\nu(\mathrm{C}-\mathrm{C}) \mathrm{R} 1[34]+\nu(\mathrm{C}-\mathrm{C}) \mathrm{BB}[6]$ \\
\hline 1463 & 1453 & $\begin{array}{l}\nu(\mathrm{C}-\mathrm{C}) \mathrm{R} 1[32]+\nu(\mathrm{C}-\mathrm{O}) \text { adj } \mathrm{R} 1[15]+\Phi(\mathrm{C}-\mathrm{C}-\mathrm{O}) \text { adj } \mathrm{R} 1 \text { link } \mathrm{O}[14] \\
\quad+\nu(\mathrm{C}-\mathrm{C}) \text { adj } \mathrm{R} 1[8]+\nu(\mathrm{C}-\mathrm{O}) \text { link } \mathrm{R} 1[8]+\Phi(\mathrm{C}-\mathrm{C}-\mathrm{O}) \text { adj } \mathrm{R} 1[7] \\
\quad+\Phi(\mathrm{C}-\mathrm{C}-\mathrm{C}) \text { adj } \mathrm{R} 1 \text { ter }[6]\end{array}$ \\
\hline 1385 & 1389 & $\begin{array}{l}\nu(\mathrm{C}-\mathrm{C}) \mathrm{cmn} \mathrm{R} 1 \& \mathrm{R} 2[48]+\nu(\mathrm{C}-\mathrm{C}) \text { adj cmn R1\&R2 [17] }+\nu(\mathrm{C}-\mathrm{C}) \mathrm{R} 1[12] \\
\quad+\Phi(\mathrm{C}-\mathrm{C}-\mathrm{C}) \mathrm{R} 1[12]+\nu(\mathrm{C}-\mathrm{O}) \text { adj } \mathrm{R} 1[12]+\nu(\mathrm{C}-\mathrm{C}) \mathrm{R} 2[5] \\
\quad+\Phi(\mathrm{C}-\mathrm{C}-\mathrm{C}) \text { adj } \mathrm{R} 2[8]\end{array}$ \\
\hline 1351 & 1351 & $\begin{array}{l}\nu(\mathrm{C}-\mathrm{C}) \text { adj } \mathrm{R} 1[37]+\nu(\mathrm{C}-\mathrm{O}) \text { adj } \mathrm{R} 1[24]+\nu(\mathrm{C}-\mathrm{C}) \mathrm{R} 1[12] \\
\quad+\Phi(\mathrm{C}-\mathrm{C}-\mathrm{C}) \mathrm{R} 1[6]+\nu(\mathrm{C}-\mathrm{C}) \text { adj } \mathrm{R} 1 \text { link } \mathrm{O}[6]\end{array}$ \\
\hline 1300 & 1297 & $\begin{array}{l}\nu(\mathrm{C}-\mathrm{C}) \mathrm{R} 1[53]+\nu(\mathrm{C}-\mathrm{O}) \text { link } \mathrm{R} 1[15]+\nu(\mathrm{C}-\mathrm{C}) \text { adj } \mathrm{R} 1 \text { link } \mathrm{O}[15] \\
\quad+\nu(\mathrm{C}-\mathrm{O}) \text { adj } \mathrm{R} 1[7]+\nu(\mathrm{C}-\mathrm{C}) \mathrm{R} 2[6]\end{array}$ \\
\hline 1278 & 1270 & $\begin{array}{l}\nu(\mathrm{C}-\mathrm{C}) \mathrm{R} 1[53]+\nu(\mathrm{C}-\mathrm{C}) \text { adj } \mathrm{cmn} \mathrm{R} 1 \& \mathrm{R} 2[14]+\nu(\mathrm{C}-\mathrm{C}) \mathrm{BB}[13] \\
\nu(\mathrm{C}-\mathrm{C}) \text { adj } \mathrm{R} 21[11]+\nu(\mathrm{C}-\mathrm{C}) \text { adj } \mathrm{R} 1[10]+\nu(\mathrm{C}-\mathrm{C}) \mathrm{cmn} \mathrm{R} 1 \& \mathrm{R} 2[7] \\
\quad+\nu(\mathrm{C}-\mathrm{C}) \mathrm{R} 2[5]\end{array}$ \\
\hline 1207 & 1207 & $\nu(\mathrm{C}-\mathrm{C}) \mathrm{R} 3[62]+\nu(\mathrm{C}-\mathrm{C})$ adj $\mathrm{R} 3[10]+\Phi(\mathrm{C}-\mathrm{C}=\mathrm{C})$ adj $\mathrm{R} 3[5]+\nu(\mathrm{C}-\mathrm{C}) \mathrm{R} 2[5]$ \\
\hline 1149 & 1158 & $\nu(\mathrm{C}-\mathrm{O})$ link $\mathrm{R} 1[38]+\nu(\mathrm{C}-\mathrm{C}) \mathrm{R} 1[34]+\nu(\mathrm{C}-\mathrm{C}) \mathrm{R} 2[16]+\nu(\mathrm{C}-\mathrm{C})$ adj $\mathrm{R} 1$ link $\mathrm{O}[6]$ \\
\hline 1139 & 1130 & $\nu(\mathrm{C}-\mathrm{C}) \mathrm{BB}[50]+\nu(\mathrm{C}-\mathrm{C}) \mathrm{R} 1[31]+\nu(\mathrm{C}-\mathrm{C}) \mathrm{R} 2[8]$ \\
\hline 1082 & 1090 & $\begin{array}{l}\nu(\mathrm{C}-\mathrm{C}) \mathrm{R} 2[45]+\nu(\mathrm{C}-\mathrm{O}) \text { link } \mathrm{R} 1[14]+\nu(\mathrm{C}-\mathrm{C}) \mathrm{R} 2[14]+\Phi(\mathrm{C}-\mathrm{C}-\mathrm{C}) \mathrm{R} 1[13] \\
\quad+\nu(\mathrm{C}-\mathrm{C}) \text { adj } \mathrm{R} 1 \text { link O [9] }\end{array}$ \\
\hline 1047 & 1038 & $\begin{array}{l}\nu(\mathrm{C}-\mathrm{O}) \mathrm{R} 3[32]+\nu(\mathrm{C}=\mathrm{C}) \mathrm{R} 3[13]+\nu(\mathrm{C}-\mathrm{C}) \text { adj } \mathrm{R} 3[12]+\Phi(\mathrm{C}-\mathrm{C}-\mathrm{C}) \mathrm{BB}[6] \\
\quad+\Phi(\mathrm{C}-\mathrm{C}-\mathrm{C}) \mathrm{R} 2 \ln \mathrm{BB}[5]\end{array}$ \\
\hline 956 & 952 & $\nu(\mathrm{C}-\mathrm{C}) \mathrm{R} 1[40]+\nu(\mathrm{C}-\mathrm{O})$ adj $\mathrm{R} 1[16]+\nu(\mathrm{C}-\mathrm{C}) \mathrm{R} 2[16]+\nu(\mathrm{C}-\mathrm{C})$ adj $\mathrm{R} 2[6]$ \\
\hline 913 & 903 & $\begin{array}{l}\nu(\mathrm{C}-\mathrm{C}) \mathrm{R} 3[22]+\nu(\mathrm{C}-\mathrm{O}) \mathrm{R} 3[18]+\Phi(\mathrm{C}-\mathrm{C}-\mathrm{C}) \mathrm{R} 2 \ln \mathrm{BB}[8] \\
\quad+\Phi(\mathrm{C}-\mathrm{C}-\mathrm{C}) \mathrm{BB}[6]+\nu(\mathrm{C}-\mathrm{O}) \mathrm{R} 3 \operatorname{adj}(=\mathrm{O})[5]\end{array}$ \\
\hline 852 & 850 & $\tau(\mathrm{C}=\mathrm{O})$ adj $\mathrm{R} 3[52]+\tau(\mathrm{C}-\mathrm{O}) \mathrm{R} 3[30]+\tau(\mathrm{C}-\mathrm{C}) \mathrm{R} 3[6]+\tau(\mathrm{C}-\mathrm{C})$ adj $\mathrm{R} 3[6]$ \\
\hline 756 & 757 & $\Phi(\mathrm{C}-\mathrm{C}-\mathrm{C}) \mathrm{R} 1[21]+\nu(\mathrm{C}-\mathrm{C}) \mathrm{R} 1[15]+\nu(\mathrm{C}-\mathrm{C}) \mathrm{R} 2[14]+\nu(\mathrm{C}-\mathrm{O})$ adj $\mathrm{R} 1$ [7] \\
\hline
\end{tabular}


Table 3

(Continued)

\begin{tabular}{|c|c|c|}
\hline $\begin{array}{l}\text { Calculated freq. } \\
\quad\left(\mathrm{cm}^{-1}\right)\end{array}$ & $\begin{array}{l}\text { Observed freq. } \\
\left(\mathrm{cm}^{-1}\right)\end{array}$ & Assignment (\% potential energy distribution) \\
\hline 714 & 713 & $\begin{array}{l}\Phi(\mathrm{C}-\mathrm{C}-\mathrm{C}) \mathrm{R} 2[20]+\nu(\mathrm{C}-\mathrm{C}) \mathrm{R} 1[10]+\Phi(\mathrm{C}-\mathrm{C}-\mathrm{C}) \text { adj } \mathrm{R} 2 \ln \mathrm{BB}[8] \\
\quad+\Phi(\mathrm{C}-\mathrm{C}-\mathrm{C}) \mathrm{R} 1[8]+\Phi(\mathrm{C}-\mathrm{C}-\mathrm{C}) \mathrm{R} 2 \ln \mathrm{BB}[7]+\Phi(\mathrm{C}-\mathrm{C}=\mathrm{C}) \text { adj } \mathrm{R} 2[7] \\
\quad+\Phi(\mathrm{C}=\mathrm{C}-\mathrm{C}) \mathrm{R} 3[6]+\Phi(\mathrm{C}-\mathrm{C}-\mathrm{O}) \mathrm{R} 3[5]+\nu(\mathrm{C}-\mathrm{C}) \mathrm{R} 2[5]\end{array}$ \\
\hline 639 & 642 & $\begin{array}{l}\Phi(\mathrm{C}-\mathrm{C}=\mathrm{C}) \text { adj } \mathrm{R} 2[29]+\nu(\mathrm{C}-\mathrm{C}) \mathrm{R} 2[20]+\Phi(\mathrm{C}-\mathrm{C}-\mathrm{C}) \mathrm{R} 1[7]+\Phi(\mathrm{C}-\mathrm{C}-\mathrm{C}) \mathrm{R} 2[6] \\
\quad+\Phi(\mathrm{O}-\mathrm{C}-\mathrm{C}) \mathrm{cmn} \mathrm{R} 1 \& \mathrm{R} 2[5]\end{array}$ \\
\hline 576 & 566 & $\begin{array}{l}\Phi(\mathrm{C}-\mathrm{C}-\mathrm{C}) \mathrm{R} 2[17]+\Phi(\mathrm{C}-\mathrm{C}=\mathrm{C}) \text { adj } \mathrm{R} 2[12]+\Phi(\mathrm{C}-\mathrm{C}-\mathrm{C}) \mathrm{R} 1[12]+\nu(\mathrm{C}-\mathrm{C}) \mathrm{R} 1[8] \\
\quad+\nu(\mathrm{C}-\mathrm{C}) \mathrm{R} 2[7]+\Phi(\mathrm{C}-\mathrm{C}-\mathrm{O}) \text { adj } \mathrm{R} 1 \ln \mathrm{O}[6]+\Phi(\mathrm{C}-\mathrm{C}-\mathrm{C}) \text { adj } \mathrm{R} 1 \ln \mathrm{O}[5]\end{array}$ \\
\hline
\end{tabular}

Abbreviations used in the table have following meanings: $\mathrm{R}$ - ring; adj - adjacent to; ln - linking; cmn - common to; $\mathrm{BB}$ - back bone.

the first time and this makes it imperative to discuss all the vibrational modes with significant potential energy distribution and hence their relation with the conformation of deoxy-andrographolide (Fig. 1). All the significant vibrational modes involving the prime sites are discussed as follows.

Rings R1, R2 and R3. Both the andrographolide and deoxy-andrographolide are diterpenes. Both have identical structure except ring R3. The andrographolide has a hydroxyl group at $\mathrm{C} 14$ and the $\mathrm{C} 16=\mathrm{O} 23$ carbonyl group is in conjugation with $\mathrm{C} 12-\mathrm{C} 13$, whereas in deoxy-andrographolide, hydroxyl group at $\mathrm{C} 14$ is absent. In place of hydroxyl group a double bond in the ring $\mathrm{R} 3$ at $\mathrm{C} 13=\mathrm{C} 14$ is present which is in conjugation with the $\mathrm{C} 16=\mathrm{O} 23$ carbonyl group. This is supported by a low potential energy distribution value (C12-C13) $28 \%$ in deoxy-andrographolide and high P.E.D. value $47 \%$ in andrographolide. The planarity of ring R3 in deoxy-andrographolide may be because of this difference which is also evidenced by $\mathrm{X}$-ray studies.

The $\mathrm{C}=\mathrm{O}$ stretching mode at $1728 \mathrm{~cm}^{-1}$ of andrographolide is comparable to $1754 \mathrm{~cm}^{-1}$ in deoxyandrographolide. The (C16-O23) 51\% in deoxy-andrographolde can be compared to (C16-O23) 71\% in andrographolide. This value can also be compared with $\mathrm{C}=\mathrm{O}$ stretch mode in gamma-butyrolactone [34] in which it appears at $1772 \mathrm{~cm}^{-1}$. This value is very typical for similar modes in lactones and identical fragments [32].

The five membered ring $\mathrm{R} 3$ has a planar orientation which was also illustrated by low value of torsion angles $(\mathrm{C} 10-\mathrm{C} 9-\mathrm{C} 11-\mathrm{C} 12),(\mathrm{C} 9-\mathrm{C} 11-\mathrm{C} 12-\mathrm{C} 13)$ and $(\mathrm{C} 11-\mathrm{C} 12-\mathrm{C} 13-\mathrm{C} 16)$ as shown by $\mathrm{X}$-ray studies also. A slightly high P.E.D. i.e. 49\% (C9-C11) in deoxy-andrographolide as compared to $44 \%$ in andrographolide may be because of this reason.

As suggested by the crystallographic study and along with our normal mode calculations it can be said that the ring $\mathrm{R} 3$ remains in a planar form and R1, R2 in chair form. The P.E.D. data further suggests that $\mathrm{C}$ attached to heteroatom i.e., $\mathrm{O}$ has high P.E.D. value $(\mathrm{C} 15-\mathrm{O} 22) 32 \%$. The low value of $(\mathrm{C} 3-\mathrm{O} 21)$ $15 \%$ as compared to $(\mathrm{C} 16-\mathrm{O} 23) 51 \%$ is suggestive of the close proximity of $(\mathrm{C} 16-\mathrm{O} 23)$ by ring junction (C12-C11) 50\% and rings R1 and R2. For the vibrational band at $639 \mathrm{~cm}^{-1}$ the normal mode calculation yields a P.E.D. with $\Phi(C-C=C) 29 \%$ whereas the deformation $\Phi(C-C-C)$ is only $7 \%$.

\subsection{Quantum mechanical Hartree-Fock calculations}

\subsubsection{Molecular geometry}

The optimized structure parameters of Deoxy-andrographolide calculated by ab initio HF level with 3-21 basis set are listed in Table 4 in accordance with the atom numbering scheme given in Fig. 1. For 


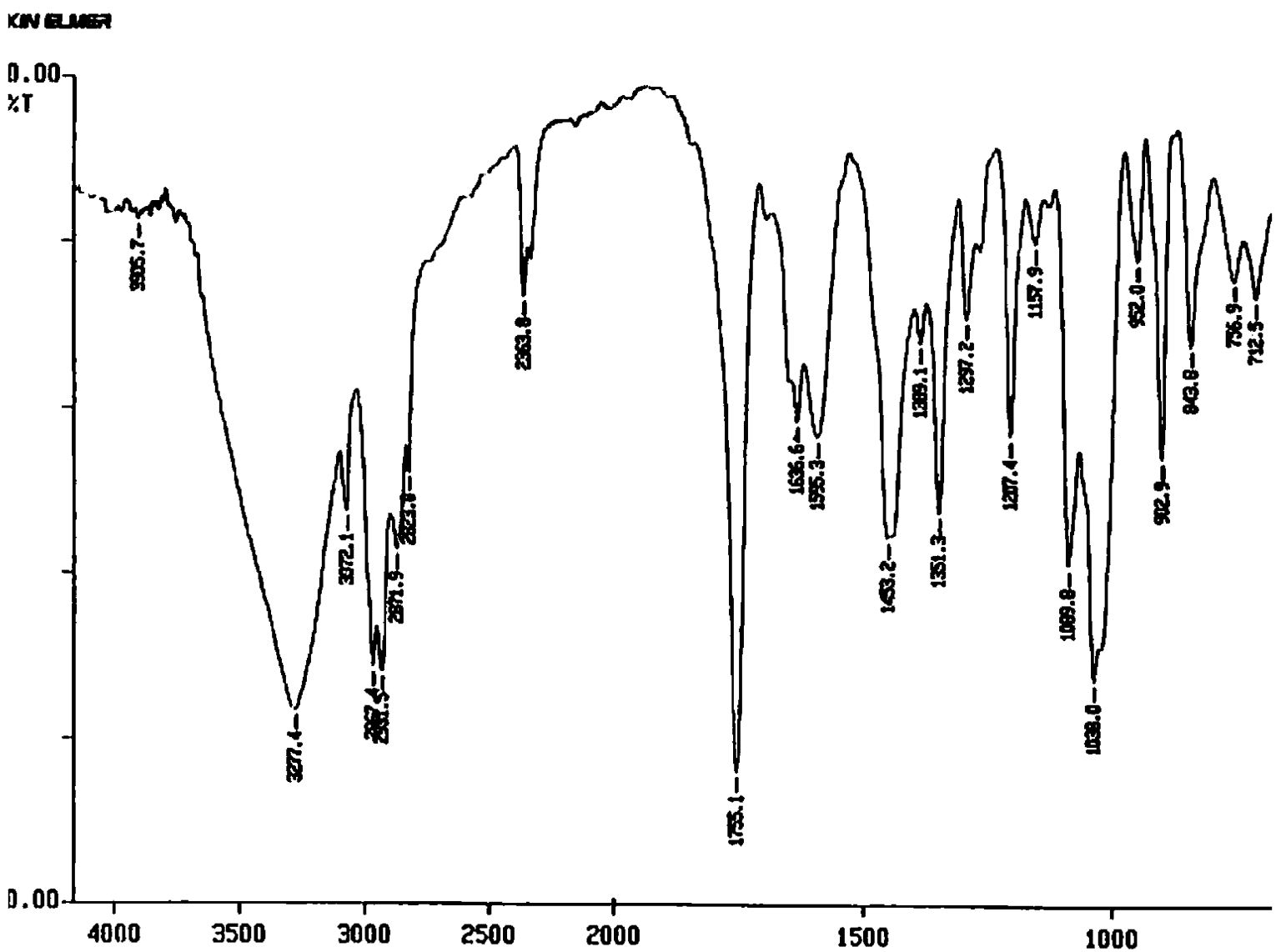

35/02/07 11:04 AH. CODE-DEAND

k: 4 scans, $4.0 \mathrm{~cm}-1$, flat, smooth, abex

Fig. 2. FT-IR spectra of Deoxyandrographolide $(4000-700) \mathrm{cm}^{-1}$.

example, the optimized bond lengths of $\mathrm{C}-\mathrm{C}$ in $\mathrm{R} 1$ ring falls in the range from $1.512 \AA$ to $1.564 \AA$ for $\mathrm{HF}$, which is in good agreement with those of experimental bond length for deoxy andrographolide (1.519$1.590 \AA$ ). The optimized bond length $\mathrm{C} 13-\mathrm{C} 14$ for $\mathrm{HF}$ is $1.317 \AA$, which is in excellent agreement with experimentally obtained bond length $1.314 \AA$. The optimized bond length $\mathrm{C} 12-\mathrm{C} 13$ involving the backbone for HF level is $1.4967 \AA$ and is in agreement with the experimentally obtained value of $1.474 \AA$. The other calculated bond lengths and bond angles also show an excellent agreement with the X-ray data. Based on above comparison it can be said that although, there are some differences between the theoretical and experimental values, the optimized structural parameters can well reproduce the experimental ones and they are the basis for thereafter discussion.

The title molecule Deoxy-andrographolide has 54 atoms. The harmonic vibrational frequencies calculated for Deoxy-andrographolide at HF level using 3-21 basis set are listed in Table 5. Comparison of the frequencies calculated at HF/3-21 level with the experimental values (Table 5) reveals the overestimation of the calculated vibrational modes due to neglect of anharmonicity in real system. Vibrational frequencies calculated at HF/3-21 level were scaled by 0.89 [35]. 
Table 4

Optimised parameters for deoxy-andrographolide

\begin{tabular}{|c|c|c|c|}
\hline \multirow[t]{2}{*}{ S. no } & \multirow[t]{2}{*}{ Parameter } & \multicolumn{2}{|c|}{ Bond length/Bond angle (angstrom/degree) } \\
\hline & & From HF-method & From X-ray data \\
\hline 1. & $\mathrm{C} 1-\mathrm{C} 2$ & 1.5359 & 1.518 \\
\hline 2. & $\mathrm{C} 1-\mathrm{C} 10$ & 1.5487 & 1.587 \\
\hline 3. & $\mathrm{C} 2-\mathrm{C} 3$ & 1.5253 & 1.494 \\
\hline 4. & $\mathrm{C} 3-\mathrm{C} 4$ & 1.5490 & 1.545 \\
\hline 5. & $\mathrm{C} 3-\mathrm{O} 21$ & 1.4354 & 1.459 \\
\hline 6. & $\mathrm{C} 4-\mathrm{C} 5$ & 1.5646 & 1.572 \\
\hline 7. & C4-C19 & 1.5363 & 1.542 \\
\hline 8. & $\mathrm{C} 4-\mathrm{C} 20$ & 1.5475 & 1.516 \\
\hline 9. & C5-C6 & 1.5437 & 1.487 \\
\hline 10. & $\mathrm{C} 5-\mathrm{C} 10$ & 1.5736 & 1.538 \\
\hline 11. & $\mathrm{C} 6-\mathrm{C} 7$ & 1.5429 & 1.553 \\
\hline 12. & $\mathrm{C} 7-\mathrm{C} 8$ & 1.5123 & 1.505 \\
\hline 13. & $\mathrm{C} 8-\mathrm{C} 9$ & 1.5246 & 1.510 \\
\hline 14. & C8-C17 & 1.3191 & 1.326 \\
\hline 15. & C9-C10 & 1.5796 & 1.590 \\
\hline 16. & C9-C11 & 1.5453 & 1.519 \\
\hline 17. & $\mathrm{C} 10-\mathrm{C} 18$ & 1.5438 & 1.503 \\
\hline 18. & $\mathrm{C} 11-\mathrm{C} 12$ & 1.5409 & 1.489 \\
\hline 19. & $\mathrm{C} 12-\mathrm{C} 13$ & 1.4967 & 1.474 \\
\hline 20. & C13-C14 & 1.3177 & 1.314 \\
\hline 21. & C13-C16 & 1.4945 & 1.483 \\
\hline 22. & C14-C15 & 1.5165 & 1.498 \\
\hline 23. & $\mathrm{C} 15-\mathrm{O} 22$ & 1.4501 & 1.450 \\
\hline 24. & $\mathrm{C} 16-\mathrm{O} 22$ & 1.3737 & 1.339 \\
\hline 25. & $\mathrm{C} 16-\mathrm{O} 23$ & 1.1948 & 1.212 \\
\hline 26. & $\mathrm{C} 19-\mathrm{O} 24$ & 1.4567 & 1.434 \\
\hline 27. & $\mathrm{C} 2-\mathrm{C} 1-\mathrm{C} 10$ & 113.1085 & 111.500 \\
\hline 28. & $\mathrm{C} 1-\mathrm{C} 2-\mathrm{C} 3$ & 110.3985 & 110.600 \\
\hline 29. & $\mathrm{C} 2-\mathrm{C} 3-\mathrm{C} 4$ & 112.2976 & 114.100 \\
\hline 30. & $\mathrm{C} 2-\mathrm{C} 3-\mathrm{O} 21$ & 110.6908 & 108.800 \\
\hline 31. & $\mathrm{C} 4-\mathrm{C} 3-\mathrm{O} 21$ & 113.0774 & 111.400 \\
\hline 32. & C3-C4-C5 & 107.8451 & 108.100 \\
\hline 33. & C3-C4-C19 & 112.0574 & 109.600 \\
\hline 34. & C3-C4-C20 & 106.7427 & 110.500 \\
\hline 35. & C5-C4-C19 & 112.5164 & 110.500 \\
\hline 36. & $\mathrm{C} 5-\mathrm{C} 4-\mathrm{C} 20$ & 109.8447 & 108.900 \\
\hline 37. & C19-C4-C20 & 107.6749 & 109.300 \\
\hline 38. & C4-C5-C6 & 113.4807 & 113.400 \\
\hline 39. & $\mathrm{C} 4-\mathrm{C} 5-\mathrm{C} 10$ & 117.383 & 119.200 \\
\hline 40. & C6-C5-C10 & 111.3546 & 110.000 \\
\hline 41. & C5-C6-C7 & 110.3601 & 111.300 \\
\hline 42. & C6-C7-C8 & 109.9293 & 111.300 \\
\hline 43. & C7-C8-C9 & 112.6020 & 114.500 \\
\hline 44. & C7-C8-C17 & 121.5129 & 121.600 \\
\hline
\end{tabular}


Table 4

(Continued)

\begin{tabular}{|c|c|c|c|}
\hline \multirow[t]{2}{*}{ S. no } & \multirow[t]{2}{*}{ Parameter } & \multicolumn{2}{|c|}{ Bond length/Bond angle (angstrom/degree) } \\
\hline & & From HF-method & From X-ray data \\
\hline 45. & $\mathrm{C} 9-\mathrm{C} 8-\mathrm{C} 17$ & 125.8381 & 123.800 \\
\hline 46. & C8-C9-C10 & 108.9368 & 108.500 \\
\hline 47. & C8-C9-C11 & 113.5949 & 114.700 \\
\hline 48. & C10-C9-C11 & 114.7701 & 113.800 \\
\hline 49. & $\mathrm{C} 1-\mathrm{C} 10-\mathrm{C} 5$ & 108.3291 & 108.300 \\
\hline 50. & $\mathrm{C} 1-\mathrm{C} 10-\mathrm{C} 9$ & 109.6595 & 107.700 \\
\hline 51. & $\mathrm{C} 1-\mathrm{C} 10-\mathrm{C} 18$ & 109.1168 & 110.400 \\
\hline 52. & $\mathrm{C} 5-\mathrm{C} 10-\mathrm{C} 9$ & 105.7242 & 106.600 \\
\hline 53. & C5-C10-C18 & 115.2493 & 115.800 \\
\hline 54. & C9-C10-C18 & 108.6396 & 107.600 \\
\hline 55. & C9-C11-C12 & 111.5229 & 113.700 \\
\hline 56. & $\mathrm{C} 11-\mathrm{C} 12-\mathrm{C} 13$ & 113.7513 & 115.500 \\
\hline 57. & $\mathrm{C} 12-\mathrm{C} 13-\mathrm{C} 14$ & 132.2940 & 133.000 \\
\hline 58. & $\mathrm{C} 12-\mathrm{C} 13-\mathrm{C} 16$ & 119.1339 & 119.500 \\
\hline 59. & $\mathrm{C} 14-\mathrm{C} 13-\mathrm{C} 16$ & 108.5721 & 107.400 \\
\hline 60. & $\mathrm{C} 13-\mathrm{C} 14-\mathrm{C} 15$ & 110.2308 & 110.400 \\
\hline 61. & $\mathrm{C} 14-\mathrm{C} 15-\mathrm{O} 22$ & 103.3744 & 103.400 \\
\hline 62. & $\mathrm{C} 13-\mathrm{C} 16-\mathrm{O} 22$ & 107.4971 & 109.100 \\
\hline 63. & $\mathrm{C} 13-\mathrm{C} 16-\mathrm{O} 23$ & 128.8962 & 128.300 \\
\hline 64. & $\mathrm{O} 22-\mathrm{C} 16-\mathrm{O} 23$ & 123.6067 & 122.500 \\
\hline 65. & $\mathrm{C} 4-\mathrm{C} 19-\mathrm{O} 24$ & 107.9357 & 112.800 \\
\hline 66. & $\mathrm{C} 15-\mathrm{O} 22-\mathrm{C} 16$ & 110.3255 & 109.600 \\
\hline 67. & $\mathrm{C} 10-\mathrm{C} 1-\mathrm{C} 2-\mathrm{C} 3$ & 59.1536 & 61.900 \\
\hline 68. & $\mathrm{C} 2-\mathrm{C} 1-\mathrm{C} 10-\mathrm{C} 5$ & -51.2418 & -51.900 \\
\hline 69. & $\mathrm{C} 1-\mathrm{C} 2-\mathrm{C} 3-\mathrm{C} 4$ & -60.8975 & -61.400 \\
\hline 70. & $\mathrm{C} 2-\mathrm{C} 3-\mathrm{C} 4-\mathrm{C} 5$ & 54.8689 & 50.600 \\
\hline 71. & C3-C4-C5-C10 & -50.5382 & -45.200 \\
\hline 72. & $\mathrm{C} 10-\mathrm{C} 5-\mathrm{C} 6-\mathrm{C} 7$ & 58.6379 & 60.500 \\
\hline 73. & $\mathrm{C} 4-\mathrm{C} 5-\mathrm{C} 10-\mathrm{C} 1$ & 48.8470 & 46.300 \\
\hline 74. & $\mathrm{C} 5-\mathrm{C} 6-\mathrm{C} 7-\mathrm{C} 8$ & -54.8664 & -51.600 \\
\hline 75. & $\mathrm{C} 6-\mathrm{C} 7-\mathrm{C} 8-\mathrm{C} 9$ & 57.8542 & 55.3501 \\
\hline 76. & C7-C8-C9-C10 & -61.9312 & -55.700 \\
\hline 77. & C8-C9-C10-C5 & 60.6032 & 60.600 \\
\hline 78. & C16-C13-C14-C15 & 0.012 & -0.300 \\
\hline 79. & $\mathrm{C} 14-\mathrm{C} 13-\mathrm{C} 16-\mathrm{O} 22$ & -0.0436 & -0.600 \\
\hline 80. & $\mathrm{C} 13-\mathrm{C} 14-\mathrm{C} 15-\mathrm{O} 22$ & 0.0215 & 1.100 \\
\hline 81. & $\mathrm{C} 14-\mathrm{C} 15-\mathrm{O} 22-\mathrm{C} 16$ & -0.0494 & -1.400 \\
\hline 82. & $\mathrm{C} 13-\mathrm{C} 16-\mathrm{O} 22-\mathrm{C} 15$ & 0.0579 & 1.300 \\
\hline
\end{tabular}

Carbonyl absorption. Carbonyl absorptions are sensitive and both the carbon and oxygen atoms of the carbonyl group move during the vibration and they have nearly equal amplitude. In the present study the $\mathrm{C}=\mathrm{O}$ stretching vibration is observed at $1755 \mathrm{~cm}^{-1}$ and is in perfect agreement with the calculated frequency obtained at $1794 \mathrm{~cm}^{-1}$.

$\mathrm{C}-\mathrm{H}$ vibrations. The hetero aromatic structure shows the presence of $\mathrm{C}-\mathrm{H}$ stretching vibrations in 
Table 5

Comparison of the observed and calculated vibrational spectra of 14-deoxyandrographolide

\begin{tabular}{|c|c|c|}
\hline $\mathrm{HF}$ & IR & Approximate mode description \\
\hline 3478 & $3905(\mathrm{vw})$ & $\nu(\mathrm{O}-\mathrm{H})$ adj $\mathrm{R} 1$ \\
\hline 3370 & 3277 (vs) & $\nu(\mathrm{O}-\mathrm{H})$ adj $\mathrm{R} 1$ \\
\hline 3058 & $3072(\mathrm{~m})$ & $\nu(\mathrm{C}-\mathrm{H}) \mathrm{R} 3$ \\
\hline 2967 & 2967 (s) & $\operatorname{sym} \nu(\mathrm{C}-\mathrm{H})$ adj $\mathrm{R} 2$ \\
\hline 2931 & $2932(\mathrm{~m})$ & $\operatorname{asym} \nu(\mathrm{C}-\mathrm{H})$ adj $\mathrm{R} 1+\operatorname{asym} \nu(\mathrm{C}-\mathrm{H}) \mathrm{R} 2$ \\
\hline 2869 & $2872(\mathrm{~m})$ & $\operatorname{sym} \nu(\mathrm{C}-\mathrm{H}) \mathrm{R} 1+\operatorname{asym} \nu(\mathrm{C}-\mathrm{H}) \mathrm{R} 2$ \\
\hline 2834 & $2364(\mathrm{~m})$ & $\nu(\mathrm{C}-\mathrm{H}) \mathrm{R} 2$ \\
\hline 1794 & 1755 (vs) & $\nu(\mathrm{C}=\mathrm{O})$ adj $\mathrm{R} 3$ \\
\hline 1665 & $1700(w)$ & $\nu(\mathrm{C}=\mathrm{C}) \mathrm{R} 3+\Phi(\mathrm{H}-\mathrm{C}-\mathrm{C})$ adj $\mathrm{R} 3$ \\
\hline 1662 & $1637(\mathrm{~m})$ & $\nu(\mathrm{C}=\mathrm{C}) \mathrm{R} 2+\operatorname{scis}(\mathrm{H}-\mathrm{C}-\mathrm{H})$ adj $\mathrm{R} 2$ \\
\hline 1523 & $1595(\mathrm{~m})$ & $\operatorname{scis}(\mathrm{H}-\mathrm{C}-\mathrm{H})$ adj $\mathrm{R} 1$ \\
\hline 1446 & $1453(\mathrm{~s})$ & $\operatorname{scis}(\mathrm{H}-\mathrm{C}-\mathrm{H})$ adj $\mathrm{R} 1$ \\
\hline 1386 & $1389(w)$ & $\Phi(\mathrm{C}-\mathrm{C}-\mathrm{H}) \mathrm{cmn} \mathrm{R} 1 \& \mathrm{R} 2+\Phi(\mathrm{C}-\mathrm{C}-\mathrm{H}) \mathrm{R} 1$ \\
\hline 1352 & $1351(\mathrm{~s})$ & $\Phi(\mathrm{H}-\mathrm{C}-\mathrm{C}) \mathrm{R} 1+$ twist $(\mathrm{H}-\mathrm{C}-\mathrm{H}) \mathrm{BB}$ \\
\hline 1297 & $1297(\mathrm{~m})$ & twist $(\mathrm{H}-\mathrm{C}-\mathrm{H}) \mathrm{BB}+\Phi(\mathrm{H}-\mathrm{C}-\mathrm{O})$ adj $\mathrm{R} 1$ \\
\hline 1274 & $1270(\mathrm{sh})$ & twist $(\mathrm{H}-\mathrm{C}-\mathrm{H})$ adj $\mathrm{R} 1+\Phi(\mathrm{H}-\mathrm{C}-\mathrm{C}) \mathrm{R} 1$ \\
\hline 1213 & $1207(\mathrm{~s})$ & $\operatorname{twist}(\mathrm{H}-\mathrm{C}-\mathrm{H}) \mathrm{R} 2+\Phi(\mathrm{H}-\mathrm{C}=\mathrm{C})$ adj $\mathrm{R} 2+\operatorname{rock}(\mathrm{H}-\mathrm{C}-\mathrm{H})$ adj $\mathrm{R} 2$ \\
\hline 1149 & $1158(\mathrm{~m})$ & $\nu(\mathrm{C}-\mathrm{O}) \mathrm{R} 3+\operatorname{twist}(\mathrm{H}-\mathrm{C}-\mathrm{H}) \mathrm{R} 2+\Phi(\mathrm{C}=\mathrm{C}-\mathrm{C}) \mathrm{R} 3$ \\
\hline 1129 & $1130(w)$ & $\operatorname{rock}(\mathrm{H}-\mathrm{C}-\mathrm{H})$ adj $\mathrm{R} 1+\Phi(\mathrm{H}-\mathrm{O}-\mathrm{C})$ adj $\mathrm{R} 1$ \\
\hline 1070 & 1090 (vs) & $\Phi(\mathrm{H}-\mathrm{C}-\mathrm{C})$ adj cmn R1\&R2 + twist $(\mathrm{H}-\mathrm{C}-\mathrm{C}) \mathrm{R} 2+\Phi(\mathrm{H}-\mathrm{C}-\mathrm{H}) \mathrm{R} 1$ \\
\hline 1038 & $1038(\mathrm{~m})$ & $\operatorname{rock}(\mathrm{H}-\mathrm{C}-\mathrm{H}) \mathrm{R} 3+\Phi(\mathrm{C}-\mathrm{C}-\mathrm{O}) \mathrm{R} 3+\Phi(\mathrm{C}-\mathrm{C}-\mathrm{C}) \mathrm{BB}$ adj $\mathrm{R} 3$ \\
\hline 946 & $952(\mathrm{~m})$ & twist $(\mathrm{H}-\mathrm{C}-\mathrm{C})$ adj $\mathrm{R} 2+\Phi(\mathrm{C}-\mathrm{C}-\mathrm{C})$ adj $\mathrm{R} 1+\Phi(\mathrm{C}-\mathrm{C}-\mathrm{C})$ adj $\mathrm{R} 1$ \\
\hline 899 & $902(\mathrm{~s})$ & def $\mathrm{R} 2+\Phi(\mathrm{C}-\mathrm{C}-\mathrm{C}) \mathrm{BB}$ adj $\mathrm{R} 2+\Phi(\mathrm{C}-\mathrm{C}-\mathrm{H}) \mathrm{R} 3$ \\
\hline 849 & $844(\mathrm{~m})$ & $\operatorname{rock}(\mathrm{H}-\mathrm{C}-\mathrm{H}) \mathrm{R} 1+$ twist $(\mathrm{H}-\mathrm{C}-\mathrm{H})$ adj $\mathrm{R} 2+$ puck R3 \\
\hline 765 & $757(w)$ & puck $\mathrm{R} 3+\operatorname{rock}(\mathrm{H}-\mathrm{C}-\mathrm{H})$ adj $\mathrm{R} 2+\tau(\mathrm{C}-\mathrm{C}-\mathrm{C}-\mathrm{C}) \mathrm{BB}$ \\
\hline 716 & $713(w)$ & $\operatorname{rock}(\mathrm{H}-\mathrm{C}-\mathrm{H})$ adj $\mathrm{R} 2+\omega(\mathrm{H}-\mathrm{C}-\mathrm{C}-\mathrm{C}) \mathrm{R} 2+\tau(\mathrm{H}-\mathrm{C}-\mathrm{C}-\mathrm{H}) \mathrm{R} 2$ \\
\hline 625 & $642(w)$ & $\operatorname{def} \mathrm{R} 3+\tau(\mathrm{C}-\mathrm{C}-\mathrm{C}-\mathrm{C}) \mathrm{BB}$ adj $\mathrm{R} 2+\omega(\mathrm{C}=\mathrm{C}-\mathrm{C}-) \mathrm{R} 2$ \\
\hline 570 & $565(w)$ & rock $\mathrm{R} 3+\Phi(\mathrm{O}=\mathrm{C}-\mathrm{O}) \mathrm{R} 3+\tau(\mathrm{H}-\mathrm{C}-\mathrm{C}-\mathrm{C}) \mathrm{R} 2$ \\
\hline
\end{tabular}

Meaning of abbreviation: $\mathrm{w}$ - weak; vs - very strong; $\mathrm{s}$ - strong; $\mathrm{m}$ - middle; sh - shoulder; $\mathrm{cmn}$ - common to; adj - adjacent to; BB - back bone; $\nu$ - stretching; $\Phi$ - bending; $\tau$ - torsion; $\omega$ - wagging; def - deformation; rock - rocking; puck - puckering; twist - twisting; scis - scissoring.

the region $2800-3000 \mathrm{~cm}^{-1}$ which is the characteristic region for the ready identification of the $\mathrm{C}-\mathrm{H}$ stretching vibration [36]. In the present study the $\mathrm{C}-\mathrm{H}$ vibrations of the title compound are observed at $2967,2872 \mathrm{~cm}^{-1}$ which is in perfect agreement with the calculated frequency at 2967 and $2869 \mathrm{~cm}^{-1}$, respectively.

$\mathrm{O}-\mathrm{H}$ vibrations. The precise position of $\mathrm{O}-\mathrm{H}$ band is dependent on the strength of hydrogen bond. The $\mathrm{O}-\mathrm{H}$ stretching is observed at $3277 \mathrm{~cm}^{-1}$ and is calculated at $3370 \mathrm{~cm}^{-1}$ in the hydrogen bonded systems. Almost in all vibrational modes overestimation is noticed at HF level due to the single wave function and the neglect of electron correlation.

\section{Conclusion}

$\mathrm{Ab}$ initio calculations using Hartree-Fock method and 3-21 basis set was performed on the title molecule for the first time with the Gaussian 03 software. The equilibrium geometries and harmonic frequen- 
cies of deoxy-andrographolide were determined and analysed at both MM and QM (HF) level of theories. The vibrational frequency calculation proved that the structure is stable (no imaginary frequencies). The difference between the observed and the calculated value of frequency of most of the fundamentals is very small. The Potential energy distribution contribution to each of the observed frequency (in MM calculations) shows the reliability and accuracy of the normal mode analysis. The normal mode analysis of deoxy-andrographolide shows a dynamical behaviour and possibly, opens up an avenue for further conformational research. With the continuing need for novel structures and the difficulty of gaining access to large tracts of bio diversity in habitats, combinatorial chemistry blended with modern quantum chemical methods can prove to be blessing for the researchers.

\section{References}

[1] G.L. Verdine, The combinatorial chemistry of nature, Nature 384 (1996), 11-13.

[2] D.R. Yance and S.M. Sagar, Targeting angiogenesis with integrative cancer therapies, Integr. Cancer Ther. 5 (2006), 4-29.

[3] X.F. Zhang and B.K. Tan, Anti-diabetic property of ethanolic extract of Andrographis paniculata in streptozocin - diabetic rats, Acta Pharmacol. Sin. 21 (2000), 1157-1164.

[4] H.Y. Zhao and W.Y. Fang, Anti-thrombotic effect of Andrographis paniculata Nees in preventing myocardial infarction, Chin. Med. J. 104 (1991), 770-775.

[5] K. Sheeja, P.K. Sheihab and G. Kuttan, Anti-oxidant and anti-inflammatory activities of the plant Andrographis paniculata Nees, Immuno. Pharm. Immunotox. 28 (2006), 129-140.

[6] P.K. Visen, B. Shukla, G.K. Patnaik and B.N. Dhawan, Andrographolide protects rat hepatocytes against paracetamol induced damage, J. Ethnopharm. 40 (1993), 131-136.

[7] C. Calabrese, S.H. Berman, J.G. Babish, X. Ma, L. Sinto, M. Dorr et al., A phase I trial of Andrographolide in HIV positive patients and normal volunteers, Phyto. Ther. Res. 14 (2000), 333-338.

[8] M.A. Hidalgo, A. Romero, J. Figuera, P. Cortes, H. Coucha, J.L. Hancke et al., Andrographolide interferes with binding of nuclear factor- $k \beta$ to DNA in HL some derived neutrophillic cells, Br. J. Chem. 21 (2002), 112-117.

[9] P.K. Singh, T. Hasan, O. Prasad, L. Sinha, K. Raj and N. Misra, FT-IR spectra and vibrational spectroscopy of Andrographolide, Spectroscopy 20 (2006), 275-283.

[10] P.K. Singh, T. Hasan, O. Prasad, L. Sinha, K. Raj and N. Misra, Vibrational spectroscopic studies and FT-IR spectra of neo-andrographolide, Spectroscopy (2007), submitted.

[11] M.A.F. Biabani, R.K. Grover, S.K. Singh, S. Kumar, K. Raj, R. Roy and B. Kundu, Tetrahedron Lett. 42 (2001), 7119.

[12] P. Thisoda, N. Rangkadilok, N. Pholphana, L. Wora suttayangkam, S. Ruchirawat and J. Satyavivad, Planta Med. 73 (2007), 503-511.

[13] R.A. Burgos, J. Monsalve, T.P. Labranche, P. Eyre and J.L. Hancke, Planta Med. 71(5) (2005), 604-608.

[14] S.R. Jada, N.H. Lajis, M.S. Saad, M.F. Stevens and J. Stanslas, Semi synthesis and cytotoxic activities of andro analogues, J. Enzym. Inhib. Med. Chem. 21(3) (2006), 327.

[15] A. Patra, A.K. Mitra, S. Biswas, C.D. Gupta, A. Basak and A.K. Barua, Carbon-13 NMR spectral studies of some labdane diterpenoids, Org. Mag. Resonance 16 (2005), 75-77.

[16] L. Cui, Q. Feng, N. Wang and Y. Xinsheng, Four new andro metabolites in human urine, Chem. Pharm. Bull. 52 (2004), 773-775.

[17] N. Misra, O. Prasad and L. Sinha, Vibrational dynamics of morphine in relation to Leu5 and Mets-enkephalins, Ind. J. Biochem. Biophys. 43 (2006), 173-181.

[18] T. Hasan, P.K. Singh, R. Misra, P. Raj and N. Misra, Vibrational dynamics of triarylantimony(v) $\mathrm{SbPh} 3[\mathrm{O} 2 \mathrm{CC}(\mathrm{OH}) \mathrm{Ph} 2] 2$, Pramana 68 (2007), 875-881.

[19] E.B. Wilson, Jr., J.C. Decius and P.C. Cross, Molecular Vibrations, Dover Publications, New York, 1980, pp. $208-239$.

[20] E.B. Wilson, Jr., A method of obtaining the expanded secular equation for the vibration frequencies of a molecule, Chem. Phys. 7 (1939), 1047-1052.

[21] E.B. Wilson, Jr., Some mathematical methods for the study of molecular vibrations, J. Chem. Phys. 9 (1941), 76-78.

[22] H.C. Urey and C.A. Bradley, The vibrations of pentatonic tetrahedral molecules, Phys. Rev. 38 (1931), 1969-1978.

[23] Rajnikant, V.K. Gupta, A. Singh, M. Lal, B.D. Gupta and B. Varghese, Crystal structure of 14-Deoxyandrographolide, Mol. Mater. 6 (1996), 227-233.

[24] A. Basak, S. Cooper, A.G. Roberge, U.K. Banik, M. Chretiens and N.G. Seidah, Inhibition of Proprotein convertases-1,7 and furin by diterpenes of Andrographis paniculata and their succinoyl esters, Biochem. J. 338 (1999), 107-113.

[25] P.L. Smith, K.N. Maloney, R.G. Pothen, J. Clardy and D.E. Clapham, Bisandro from AP activates TRPV4 Channels, J. Biol. Chem. 281 (2006), 29897-29904. 
[26] M.J. Frisch, G.W. Trucks, H.B. Schlegel, G.E. Scuseria, M.A. Robb, J.R. Cheeseman, J.A. Montgomery, Jr., T. Vreven, K.N. Kudin, J.C. Burant, J.M. Millam, S.S. Iyengar, J. Tomasi, V. Barone, B. Mennucci, M. Cossi, G. Scalmani, N. Rega, G.A. Petersson, H. Nakatsuji, M. Hada, M. Ehara, K. Toyota, R. Fukuda, J. Hasegawa, M. Ishida, T. Nakajima, Y. Honda, O. Kitao, H. Nakai, M. Klene, X. Li, J.E. Knox, H.P. Hratchian, J.B. Cross, C. Adamo, J. Jaramillo, R. Gomperts, R.E. Stratmann, O. Yazyev, A.J. Austin, R. Cammi, C. Pomelli, J.W. Ochterski, P.Y. Ayala, K. Morokuma, G.A. Voth, P. Salvador, J.J. Dannenberg, V.G. Zakrzewski, S. Dapprich, A.D. Daniels, M.C. Strain, O. Farkas, D.K. Malick, A.D. Rabuck, K. Raghavachari, J.B. Foresman, J.V. Ortiz, Q. Cui, A.G. Baboul, S. Clifford, J. Cioslowski, B.B. Stefanov, G. Liu, A. Liashenko, P. Piskorz, I. Komaromi, R.L. Martin, D.J. Fox, T. Keith, M.A. Al-Laham, C.Y. Peng, A. Nanayakkara, M. Challacombe, P.M.W. Gill, B. Johnson, W. Chen, M.W. Wong, C. Gonzalez and J.A. Pople, Gaussian Inc., Wallingford, CT, 2004.

[27] H.B. Schlegel, J. Comput. Chem. 3 (1982), 214.

[28] A. Frisch, A.B. Nielson and A.J. Holder, Gaussview User Manual, Gaussian Inc., Pittsburgh, PA, 2000.

[29] S. Gupta, M.A. Chaudhary, J.N.S. Yadav, V. Srivastava and J.S. Tandon, Anti-diarrheal activity of diterpenes of Andrographis paniculata (Kal-megh) against Escheritia coli enterotoxin in in vivo models, Int. J. Crud. Drug. Res. 28(4) (1990), 273.

[30] C. Hu, B. Zhao and Z. Caoyo, Spectrometric identification of diterpene-andrographolide, CA 531 (1981), 9720702m.

[31] T. Shimanouchi, J. Chem. Phys. 17 (1949), 245.

[32] L.X. Chan, G.X. Qu and F. Qiu, Studies on diterpenoids from Andrographis paniculata, Zhongguo Zhong Yao Zhi 31 (2006), 1594-1597.

[33] D.N. Butcher, J.D. Connolly, An investigation of factors which influence the production of abnormal terpenoids by callus cultures of Andrographis paniculata Nees, J. Exp. Bot. 22 (1971), 314-322.

[34] D.P. Mcdermott, Vibrational assignments and normal coordinate analyses of gamma-butyrlactone and 2-pyrrolodinones, J. Phys. Chem. 90 (1986), 2569.

[35] A.P. Scott and L. Radom, Harmonic vibrational frequencies: An evaluation of Hartree-Fock, Møller-Plesset, quadratic configuration interaction, density functional theory, and semiempirical scale factors, J. Phys. Chem. 100 (1996), 1650216513.

[36] V. Krishnakumar and R.J. Xavier, Ind. J. Pure Appl. Phys. 41 (2003), 597. 


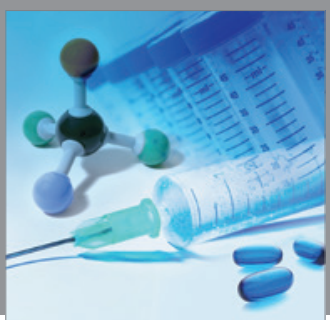

International Journal of

Medicinal Chemistry

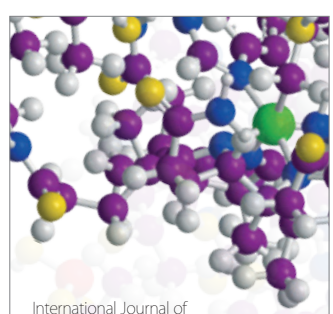

Carbohydrate Chemistry

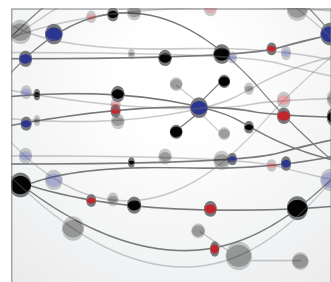

The Scientific World Journal
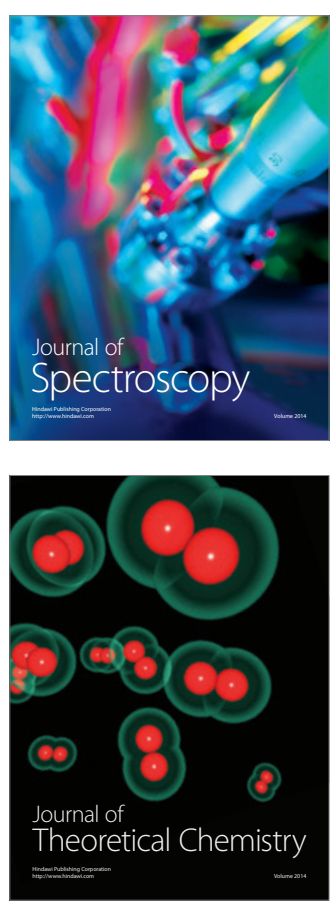
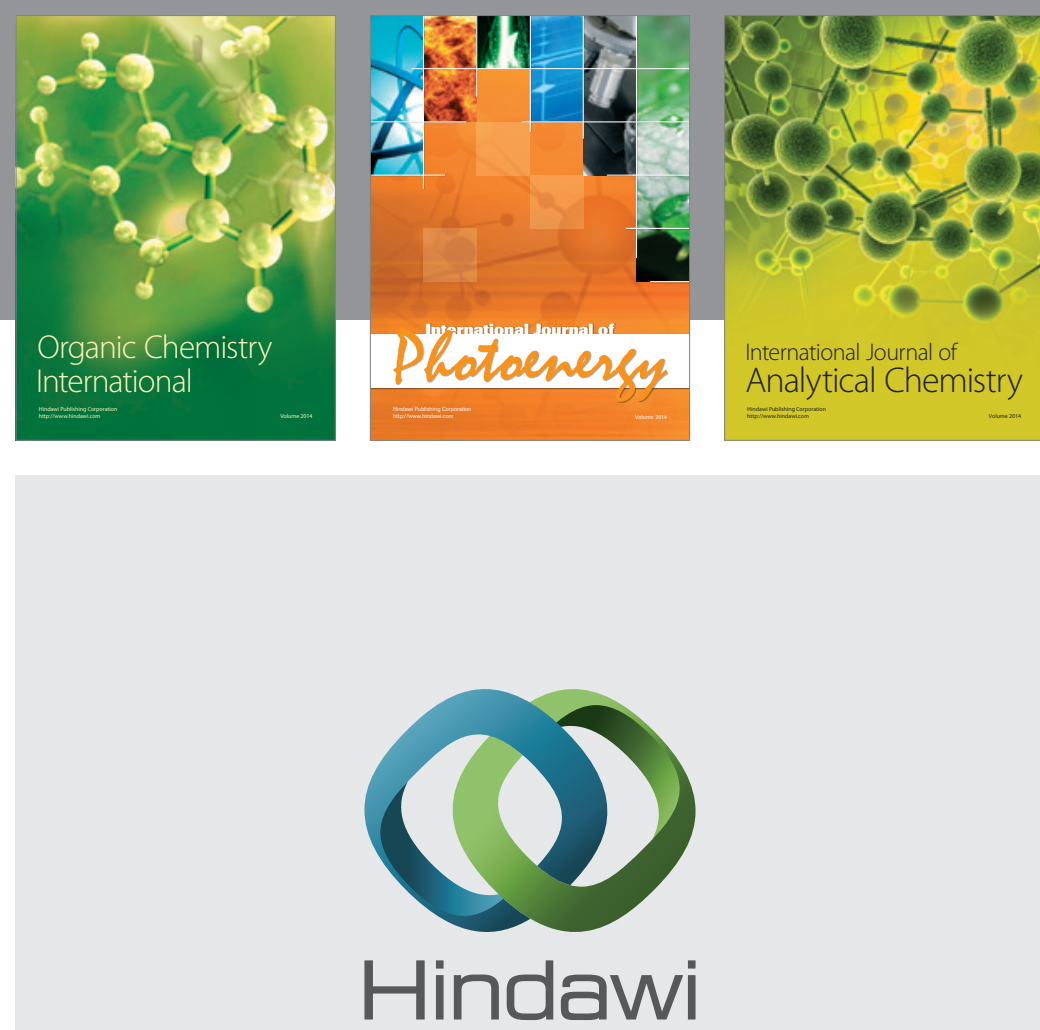

Submit your manuscripts at

http://www.hindawi.com
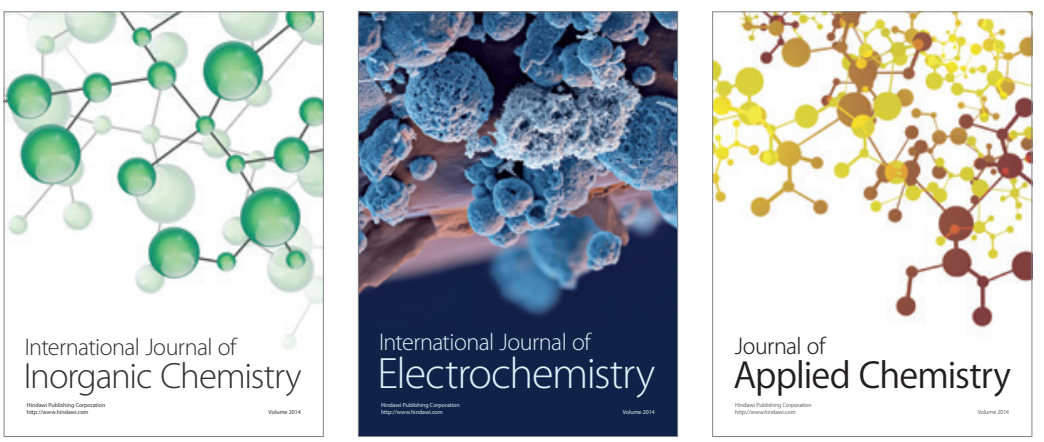

Journal of

Applied Chemistry
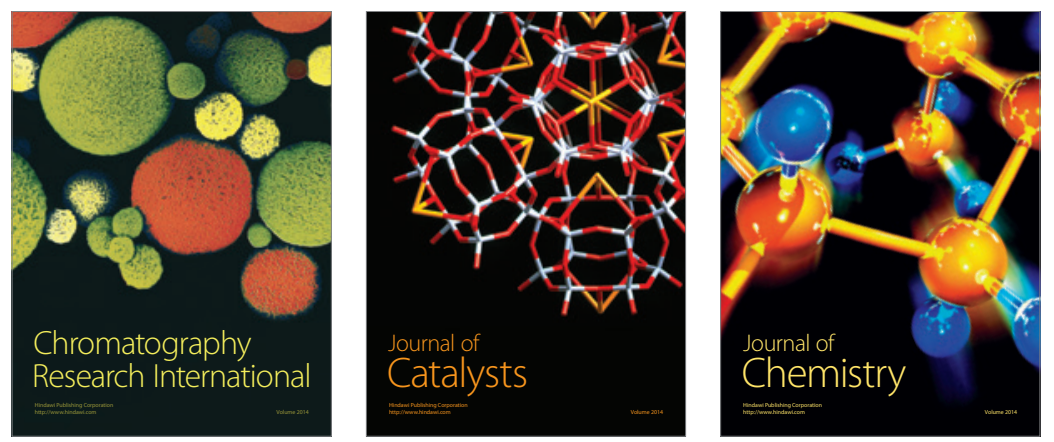
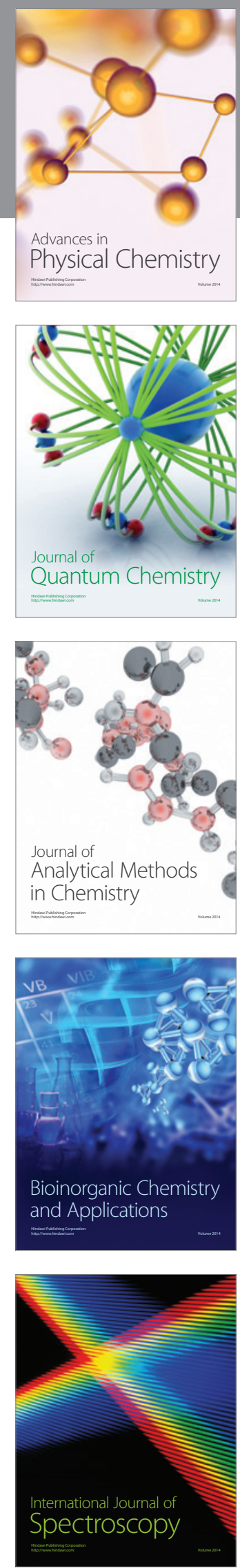